\section{Regards sur l'économie allemande}

Bulletin économique du CIRAC

$102 \mid 2011$

Varia

\title{
Médias et communication
}

FORSTER R., PETZOLD V., Im Schatten der DEFA. Private

Filmproduzenten in der DDR / FROMME J., ISKE S., MAROTZKI W. (eds), Medialität und Realität. Zur konstitutiven Kraft der Medien / HEPP A., BOZDAG C., SUNA L. Mediale Migranten. Mediatisierung und die kommunikative Vernetzung der Diaspora / JARREN O., DONGES P., Politische Kommunikation in der Mediengesellschaft. Eine Einführung / KORTE K.-R. (ed), Die Bundestagswahl 2009. Analysen der Wahl-, Parteien-, Kommunikations- und Regierungsforschung / RÜHL M., Journalistik und Journalismus im Wandel. Eine kommunikationswissenschaftliche Perspektive / SARCINELLI U., Politische Kommunikation in Deutschland. Medien und Politikvermittlung im demokratischen System / SCHULZ W., Politische Kommunikation. Theoretische Ansätze und empirische Forschung / ZAG R., Der Publikumsvertrag. Drehbuch, Emotion und der „human factor“ / ENDEWELD M., France Télévisions [off the record] / KELLER A., Der neue Rechtsrahmen für den öffentlichen Rundfunk in Frankreich / WROBELLEIPOLD A., Warum gibt es die Bild-Zeitung nicht auf Französisch? Zu Gegenwart und Geschichte der tagesaktuellen Medien in Frankreich

\footnotetext{
(2) OpenEdition

12 Journals

Édition électronique

URL : http://journals.openedition.org/rea/4338

DOI : $10.4000 /$ rea. 4338

ISBN : 978-2-8218-1134-8

ISSN : $1965-0787$

Éditeur

CIRAC

Édition imprimée

Date de publication : 31 octobre 2011

ISSN : 1156-8992

Référence électronique

"Médias et communication », Regards sur l'économie allemande [En ligne], 102 | octobre 2011, mis en ligne le 04 novembre 2011, consulté le 22 septembre 2020. URL : http://journals.openedition.org/rea/ 4338 ; DOI : https://doi.org/10.4000/rea.4338
}

Ce document a été généré automatiquement le 22 septembre 2020. 


\section{Médias et communication}

FORSTER R., PETZOLD V., Im Schatten der DEFA. Private

Filmproduzenten in der DDR / FROMME J., ISKE S., MAROTZKI W. (eds), Medialität und Realität. Zur konstitutiven Kraft der Medien / HEPP A., BOZDAG C., SUNA L. Mediale Migranten. Mediatisierung und die kommunikative Vernetzung der Diaspora / JARREN O., DONGES P., Politische Kommunikation in der Mediengesellschaft. Eine Einführung / KORTE K.-R. (ed), Die Bundestagswahl 2009. Analysen der Wahl-, Parteien-, Kommunikations- und Regierungsforschung / RÜHL M., Journalistik und Journalismus im Wandel. Eine kommunikationswissenschaftliche Perspektive / SARCINELLI U., Politische Kommunikation in Deutschland. Medien und Politikvermittlung im demokratischen System / SCHULZ W., Politische Kommunikation. Theoretische Ansätze und empirische Forschung / ZAG R., Der Publikumsvertrag. Drehbuch, Emotion und der "human factor" / ENDEWELD M., France Télévisions [off the record] / KELLER A., Der neue Rechtsrahmen für den öffentlichen Rundfunk in Frankreich / WROBELLEIPOLD A., Warum gibt es die Bild-Zeitung nicht auf Französisch? Zu Gegenwart und Geschichte der tagesaktuellen Medien in Frankreich

\section{RÉFÉRENCE}

FORSTER R., PETZOLD V., Im Schatten der DEFA. Private Filmproduzenten in der DDR, UVK Verlagsgesellschaft, Constance, 2010, 424 p.

FROMME J., ISKE S., MAROTZKI W. (eds), Medialität und Realität. Zur konstitutiven Kraft der Medien, VS Verlag für Sozialwissenschaften, Wiesbaden, 2011, 220 p. HEPP A., BOZDAG C., SUNA L. Mediale Migranten. Mediatisierung und die kommunikative Vernetzung der Diaspora, VS Verlag für Sozialwissenschaften, Wiesbaden, 2011, $290 \mathrm{p}$ JARREN O., DONGES P., Politische Kommunikation in der Mediengesellschaft. Eine Einführung, VS Verlag für Sozialwissenschaften, Wiesbaden, $3^{\mathrm{e}}$ éd.revue et augmentée, $2011,284 \mathrm{p}$ 
KORTE K.-R. (ed), Die Bundestagswahl 2009. Analysen der Wahl-, Parteien-, Kommunikations- und Regierungsforschung, VS Verlag für Sozialwissenschaften, Wiesbaden, 2010, 412 p. RÜHL M., Journalistik und Journalismus im Wandel. Eine kommunikationswissenschaftliche Perspektive, VS Verlag für Sozialwissenschaften, Wiesbaden, 2011, 272 p.

SARCINELLI U., Politische Kommunikation in Deutschland. Medien und

Politikvermittlung im demokratischen System, $3^{\mathrm{e}}$ éd. revue et augmentée, VS Verlag für Sozialwissenschaften, Wiesbaden, 2011, 408 p.

SCHULZ W., Politische Kommunikation. Theoretische Ansätze und empirische Forschung, $3^{\mathrm{e}}$ édition, VS Verlag für Sozialwissenschaften, Wiesbaden, 2011, $388 \mathrm{p}$. ZAG R., Der Publikumsvertrag. Drehbuch, Emotion und der „human factor“, UVK Verlagsgesellschaft, Constance, $2^{\mathrm{e}}$ éd. revue et augmentée, 2010, 200 p.

ENDEWELD M., France Télévisions [off the record], Flammarion, Paris, 2010, 478 p. KELLER A., Der neue Rechtsrahmen für den öffentlichen Rundfunk in Frankreich, Coll. Schriften zum internationalen und zum öffentlichen Recht, vol. 93, Peter Lang, Francfort/Main, 2011, 450 p.

WROBEL-LEIPOLD A., Warum gibt es die Bild-Zeitung nicht auf Französisch? Zu Gegenwart und Geschichte der tagesaktuellen Medien in Frankreich, VS Verlag für Sozialwissenschaften, Wiesbaden, 2010,170 p.

1 La production en sciences de la communication ou en analyse des médias est extrêmement abondante outre-Rhin. Les travaux portent d'une part sur le cinéma : FORSTER et al. nous révèlent que la production cinématographique de la RDA ne se limite nullement aux studios de la DEFA, mais qu'au contraire existaient deux bonnes douzaines de sociétés privées dont ils retracent ici l'histoire. Dans son manuel pratique du scénariste, Roland ZAG se concentre sur ce 'human factor' garant de charge émotionnelle et nous livre quelques recettes de succès auprès du public.

2 Deux autres ouvrages se concentrent sur la question du rôle des médias dans la socialisation : trois spécialistes des sciences de l'éducation (FROMME et al.) réunissent les contributions d'un colloque organisé en octobre 2009 à Magdeburg et d'un cycle de conférences qui passent en revue les différentes théories sur la force identitaire constitutive des médias, de leur apport à la formation culturelle, comme de leur impact sur l'approche de l'éducation, sans oublier la question auxquels les Allemands accordent une importance particulière : la compétence des individus en matière de médias. Trois spécialistes des sciences de la communication (HEPP et al.), se penchent sur la problématique du rôle des médias (y compris les réseaux sociaux) dans la construction identitaire des immigrés et remettent en question la politique établie d'intégration grâce aux médias.

3 Trois autres encore sont des éditions revues et augmentées de manuels de communication politique - des classiques. Le premier (JARREN/DONGES) est un manuel d'initiation aux principales théories, très clairement conçu et facilement abordable. Le grand classique de SARCINELLI, qui implique la maîtrise des connaissances de base, est centré sur le lien entre démocratie et information, ainsi que l'interaction de leurs modes d'organisation. Le manuel de SCHULZ, enfin, est plus orienté sur la recherche empirique et les liens entre politique et mass media dans la praxis, ainsi que l'évolution de la participation citoyenne. 
4 Ont peut décliner ces approches à la lecture de l'analyse des élections au Bundestag en 2009, notamment sous l'angle de la communication politique, publiée par KORTE, autre spécialiste de renom. Et à celle de l'évolution des pratiques journalistiques comme des théories américaines et européennes afférentes (RÜHL).

5 L'observation des médias étrangers est une préoccupation très développée aussi outreRhin. L'intérêt récent pour les médias français a été aiguisé, bien sûr par le dernier rapport de Reporters sans frontières, mais aussi par une série d'essais publiés en France (comme cet ouvrage très documenté d'ENDEWELD sur les liens d'influence entre TV et monde politique), et soulève régulièrement des interrogations sur la liberté des médias chez le principal partenaire de la RFA dans l'UE. A preuve ce petit ouvrage critique de WROBEL-LEIPOLD qui montre le profond étonnement des Allemands face à un système médiatique dont ils peinent d'autant plus à comprendre les subtilités que la liberté des médias n'est pas ancrée dans la Constitution. La thèse de KELLER sur les récentes évolutions du droit de l'audiovisuel vient en partie combler ces lacunes. Ce travail est d'autant plus encourageant qu'il s'agit d'une cotutelle et que, dès lors, d'autres suivront, ce qui permettra aux juristes français et allemands de se livrer à un échange plus approfondi sur les deux systèmes les plus antinomiques qui soient dans l'espace européen. (ib) 